Rakenteiden Mekaniikka (Journal of Structural Mechanics)

Vol. 51, No 1, 2018, pp. 1-9

http://rakenteidenmekaniikka.journal.fi/index

https:/doi.org/10.23998/rm.65175

CAuthor(s) 2018.

Open access under CC BY-SA 4.0 license.

\title{
Combination of permanent and variable loads
}

\author{
Tuomo Poutanen ${ }^{1}$, Sampsa Pursiainen, Jari Mäkinen and Tim Länsivaara
}

\begin{abstract}
Summary. This paper concentrates on the combination of permanent and variable loads in the structural probability theory and its implementation in codes. In the current codes, the permanent and variable loads are sometimes combined uncorrelated, and sometimes they are combined correlated. We propose that, for the safe outcome in the standardized load estimation, the actual permanent and variable loads should be combined correlated without any load reduction. The load reduction arising from the uncorrelated combination leads to an unsafe design. For example, when the permanent and variable loads are both equal to 1, the combination load is 2 if the correlated combination is applied. However, the value predicted by the model for uncorrelated load combination is less and for example only ca 1.86 in one uncorrelated load combination option of the Eurocodes. Although the load formation processes of the load pairs are uncorrelated, the correlated combination is applied since the load formation and the load combination are different processes. To support our view, we present arguments and examples based on probability theory, physics and statics and relate them with the current codes.
\end{abstract}

Key words: permanent load, variable load, load combination, code, reliability

Received 7 October 2017. Accepted 26 February 2018. Published online 16 August 2018.

\section{Introduction}

This paper concerns the structural probability theory and, in particular, the load combination of permanent and variable loads, which is a basic, routinely-performed procedure in structural design. We focus on the standardized load combination and use the Eurocodes as an example where the correlated load combination, namely rule (6.10) and the uncorrelated combination, rules $(6.10 \mathrm{a}-\mathrm{b}),(6.10 \mathrm{a}$-modified-b) are presented. The latter two are based on the hypothesis that both the permanent and variable loads are uncorrelated and random and, therefore, they are combined uncorrelated. Since the load formation and the load combination are different processes, uncorrelated loads, however, do not automatically result in an uncorrelated combination. To distinguish between these two fundamental approaches to load combination, we introduce the following terms:

- Uncorrelated load combination: Two loads are combined assuming that they are uncorrelated and random. When the Monte Carlo simulation is used, the combined load distribution is computed by giving each distribution its own seed number. In this combination, one or both of the loads are multiplied with a reduction factor before

\footnotetext{
${ }^{1}$ Corresponding author: tuomo.poutanen@tut.fi
} 
calculating their sum, which decreases the estimated value for the total load. We may call this combination the 'reduced' or 'vanishing' combination, as in this combination a reduction factor is applied in one way or another, which results in a load reduction. This combination may also be called a 'non-simultaneous combination', as the design load values are non-simultaneous and either one or both loads are multiplied with a reduction factor before adding the loads together. The authors have called this combination independent combination in the earlier papers $[5,6]$

- Correlated load combination: In this approach, the two design load values are added together assuming that they occur simultaneously, the Monte Carlo simulation is conducted using one seed number, i.e., the same seed number for both loads. This combination can be characterized as a full combination, as there is no reduction or vanishing. The authors have called this combination dependent combination in the earlier papers $[5,6]$

In this paper we propose that, for the appropriate results in the standardized load combination estimation, the permanent load and the main variable load should be combined correlated. In support of this proposal we present and justify the following three arguments. (1) The loads should be added together without utilising the reduction factor that is included in the uncorrelated combination. (2) The uncorrelated load combination results in load vanishing and a higher failure probability than intended; the failure probability is higher in the combination load than in the single load. (3) This observation might help to resolve some contradictions related to load combination in the present code standards.

This article is structured as follows. Firstly, we briefly review the theoretical background for load combination and the reasoning behind the present concepts of uncorrelated and correlated load combination. Secondly, we explain the arguments (1) - (3) in separate sections based on the viewpoints of probability theory and physics. Finally, the outcome is discussed and reflected on according to the current code standards and we present our conclusions.

\section{The load combination formulas}

We begin with a brief review of how the permanent load $G, G\left(x ; \mu_{G}, \sigma_{G}\right)$ and the variable load $Q, Q\left(x ; \mu_{Q}, \sigma_{Q}\right)$ are combined. This can be done uncorrelated, semi-correlated or correlated, although the semi-correlated combination is omitted here as it is not applied in the current codes. The two other options are explained in detail below. It is important to emphasize that both the correlated and the uncorrelated approach are applied in the present codes.

\section{The uncorrelated load combination}

The uncorrelated combination distribution $G Q(x)$ can be obtained by using dedicated algorithms [2, 3, 7], the Monte Carlo simulation with two seed numbers, or by using the convolution equation $[4,5,6]$ given by the integral

$$
G Q_{i}(x)=\int_{-\infty}^{\infty} G_{d}\left(x-r ; \mu_{G}, \sigma_{G}\right) \cdot Q_{c}\left(r ; \mu_{Q}, \sigma_{Q}\right) d r,
$$


where $G Q_{i}$ is the cumulative distribution of the combination load, $G_{d}$ is the density distribution of $G$, and $Q_{c}$ is the cumulative distribution of $Q$. Formula (1) can be written in many alternative ways with the same result. If the partial distributions are normal, the uncorrelated combination distribution $G Q_{i}\left(x ; \mu_{G Q}, \sigma_{G Q}\right)$ is also normal and the following simple formula can be applied $[4,6]$ :

$$
G Q_{i}\left(x ; \mu_{G}+\mu_{Q}, \sqrt{\sigma_{G}^{2}+\sigma_{Q}^{2}}\right) .
$$

In the code standards, for the sake of simplicity, the uncorrelated combination is obtained through the sum of the component loads multiplied with a reduction factor. Normally, two permanent load factors are used.

The uncorrelated load combination is illustrated in Figure 1. Due to the boundary condition, formulas (5) and (6) are omitted here, so the total combined load is approximately $10 \%$ less than the sum of the actual loads, i.e., a part of the load vanishes.

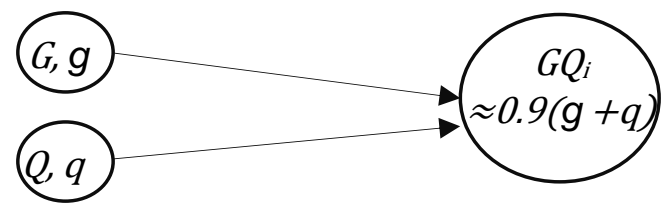

Figure 1. Illustration of the uncorrelated load combination. In this model the load formation and the load combination is one process. The combination distribution $G Q_{i}$ is constructed from the partial distributions $G$ and $Q$ using a stochastic process. The physical boundary, formulas (5) and (6), is omitted so about $10 \%$ of the load vanishes.

\section{The correlated load combination}

Loads can be combined correlated, either by using the Monte Carlo simulation and one seed number or by adding up the partial distributions by fractiles [4, 6]

$$
x_{G Q, i}=x_{G, i}+x_{Q, i}
$$

where $x_{G, i}$ and $x_{Q, i}$ are the partial loads in fractile $i$, and $x_{G Q, i}$ is the combination load in the same fractile. If the partial distributions are normal, the correlated combination distribution is also normal, $G Q_{d}\left(x ; \mu_{G Q}, \sigma_{G Q}\right)$ and one can apply the following simple formula, which can also be confirmed via a Monte Carlo simulation, [4, 6]:

$$
G Q_{d}\left(x ; \mu_{G}+\mu_{Q}, \sigma_{G}+\sigma_{Q}\right) .
$$

The correlated load combination is illustrated in Figure 2. Each load pair, $g, q$, is random in the formation process, but since they are assumed to occur simultaneously, these random loads must be combined correlated in order to satisfy the formulae (5) and (6). 


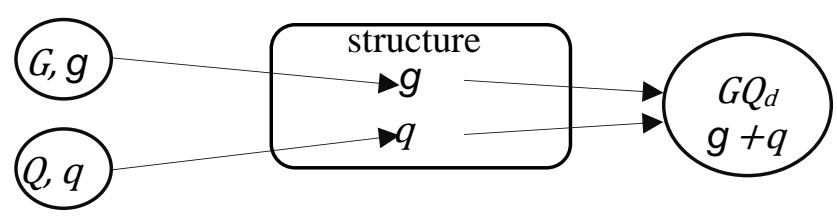

Figure 2. Illustration of the correlated load combination. The load formation and the load combination are different processes. The random loads $g$ and $q$ are here uncorrelated, but in the combination they are simultaneous and correlated.

\section{Reasons for using the correlated load combination}

This section explains the following arguments, which support using the correlated load combination approach in the standardized procedures. We propose: that the loads must be added up without a reduction factor; that the uncorrelated load combination results in load vanishing; that this observation helps to resolve some contradictions in standardized load combination and, further; that the uncorrelated combination means correlation of the loads. We base our arguments on the laws of physics and statics, and also on an example comparison of the correlated and uncorrelated load combination.

\section{The physical boundary}

In any load combination there is a boundary. This comes from the governing laws of physics and statics, and applies to all load combinations regardless of the load correlation. The basic load combination equation in the structural design is

$$
g+q=g q
$$

where $g$ is the characteristic value of the permanent load obtained from the load table of the actual material. This is normally the $50 \%$ fractile of the distribution. The value of $q$ is the characteristic value of the variable load, obtained from the load table for the actual load. It is normally the "50-year return load", i.e., the $98 \%$ fractile of the one-year distribution. The term on the right-hand side of the equation, $g q$, is the combined load with a suitable combination of different loads. The expression (5) is valid in all the design codes used in serviceability design, and also in the working stress codes of the failure design. Many new codes, e.g. the Eurocodes, are partial safety-factor codes, where the characteristic values are multiplied by safety factors in the failure design. With the safety factors $\gamma_{g}$ and $\gamma_{q}$ for the permanent and variable loads, respectively, formula (5) becomes

$$
\gamma_{g} g+\gamma_{q} q=g q
$$

In statics, the loads are combined according to Equations (5) and (6). The actions in the actual structure caused by the permanent load, $g$ or $\gamma_{g} g$ and the variable load, $q$ or $\gamma_{q} q$, are uncorrelated and are added together using the rules of statics, regardless of the correlation of the loads.

In the uncorrelated load combination, Equations (5) and (6) are, however, not exact, as a part of the load "vanishes" in the combination. This 'load vanishing' is imaginary, but it has certain consequences as are shown below. This is demonstrated in the 
uncorrelated load combination used in the Finnish code [1], i.e., the uncorrelated Eurocode combination rules (6.10a, modified-b) of the form

$$
\max (1.35 \cdot g, 1.15 \cdot g+1.5 \cdot q)=g q_{i} .
$$

In the Eurocodes, the load combination has been formulated in a rather complicated way, but it has been explained in a simple form in [8], where this combination is given in Expression (iii) on page 14. It should be noted that the Eurocodes do not employ the concept of correlated and uncorrelated load combinations as they are applied in this article. The partial factor of the permanent load value, 1.15, is due to the reduction factor $\xi$ for permanent loads in the expression (6.10b) [1].

In the Eurocodes [1], the correlated load combination, rule (6.10), or Expression (i) on page 14 of [8] is given by

$$
1.35 \cdot g+1.5 \cdot q=g q_{d} .
$$

The designer can choose to use either rule (7) or (8) for the load combination. We can conclude that the correlated combination approach (8) is formally correct. When the permanent load acts exclusively, the failure load is $1.35 \cdot \mathrm{g}$, and correspondingly, when the variable load acts exclusively, the failure load is $1.5 \cdot q$. When these loads act together, statics determine that the combination load is the sum of these loads according to formula (8). It is not possible for the increased variable load to influence the behaviour of the permanent load without losing the modelling precision. For instance, it is not realistic to assume that the increased snow load on a roof girder decreases either the permanent load of the girder or its uncertainty, which would be the consequence if formula (7) were used.

Further support for the correlated load combination is provided by the following example. Table 1 includes the correlated $g q_{d}$ and the uncorrelated $g q_{i}$ load combinations in the failure design according to formulae (7) and (8), when $g=1$ and $q=0,0.1,0.5$ and 1 respectively. We find that in the second combination, $g=1, q=0.1$, the uncorrelated combination load is $g q_{i}=1.35$. This is the same as in the first combination, with $g=1$ and $q=0$. Although the load increases, the combination load does not increase, which means that the probability of a failure remains the same. Thus, we can consider this as an inexact rule for load combination. In this case, the vanished load in comparison to the correlated combination is $10 \%$. An analogous vanishing occurs in the load combination $(6.10 \mathrm{a}-\mathrm{b})$ of the Eurocodes. This vanishing is even greater when the rule $(6.10 \mathrm{a}$, modifiedb) is used, as it often is due to its lower computational cost. However, the rule (6.10), i.e. formula (8), is much simpler in terms of calculation than either of the uncorrelated combinations.

If two permanent load factors are applied to combine the permanent and variable loads, following the combination rules (6.10a-b) and (6.10a, modified-b) of the Eurocodes, these factors include a reduction factor which results in load vanishing, i.e., an inexact combination.

The fixed and deterministic values of $g$ and $q$ obtained from the load tables and Equations (7) and (8) correspond to the load combination in the actual design. However, we have found that the uncorrelated combination rule (7) yields a contradiction in that, although there is an increase in the loading, the probability for failure remains the same. 
Table 1. Uncorrelated load combinations of the Finnish Eurocodes [1], the rule (6.10a, modified-b) and correlated combinations, the rule (6.10). $g=$ characteristic permanent load, $q=$ characteristic variable load, $g q=$ characteristic total load, $g q_{d}=$ design load for correlated combination, $g q_{i}=$ design load for uncorrelated combination.

\begin{tabular}{cccccccc}
\hline Number & $g$ & $q$ & $g q$ & $g q_{d}$ & $g q_{i}$ & $g q_{d}-g q_{i}$ & Error \% \\
\hline 1 & 1 & 0 & 1 & 1.35 & 1.35 & 0.00 & 0 \\
2 & 1 & 0.1 & 1.1 & 1.50 & 1.35 & 0.15 & 10 \\
3 & 1 & 0.5 & 1.5 & 2.10 & 1.90 & 0.20 & 10 \\
4 & 1 & 1 & 2 & 2.85 & 2.65 & 0.20 & 7 \\
\hline
\end{tabular}

\section{Column example}

Below, we give an example of the load combination for two short columns. The terms and symbols of the Eurocodes [1] are used. The design point, i.e. the characteristic value of the distributions, is set at unity. The design service life is assumed to be 50 years. The target reliability is $\beta_{50}=3.5$, i.e., the failure probability is $P_{f 50}=1 / 4300$, and the permanent and the 50-year variable load distributions are set to be normal with equal parameters: $G\left(x ; \mu_{G}, \sigma_{G}\right), V_{G}=0.1, \mu_{G}=1, \sigma_{G}=0.1$ and $Q\left(x ; \mu_{Q}, \sigma_{Q}\right), V_{Q}=0.1, \mu_{Q}=1$, $\sigma_{Q}=0.1$.

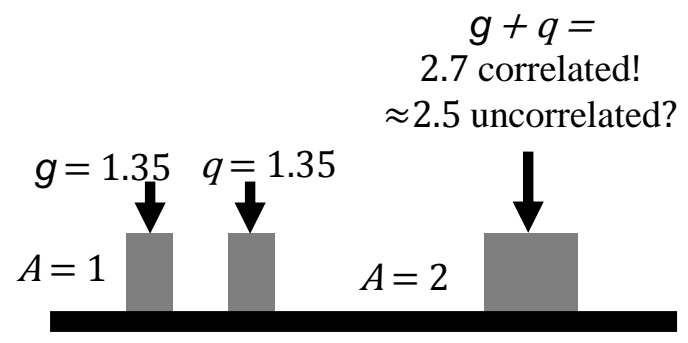

Figure 3. Illustration of the load combination. The loads $g, q$ are random in the formation but deterministic and correlated with each other in the combination.

The columns studied are illustrated in Figure 3. The one on the left is loaded with the permanent load, $G$, and the one next to it with the variable load, $Q$. The loads are assumed to be equal, $g=q=1.35$, and the material is set to be ideal, i.e., $V_{M}=0, \mu_{M}=1.35$, $\sigma_{M}=0$. Both columns fail at this load with $\beta_{50}=3.5$.

The first (physical) column supports a permanent load of 1.35 , failing at $\beta_{50}=3.5$, while the other column, which is physically equal to the first one, is added to the structure next to the first column, meaning that a variable load 1.35, e.g. a snow load, is added. Due to symmetry and statics, the columns are equally loaded with 1.35 and $\beta_{50}=3.5$. Thus, the total combination load, is 2.7 and $\beta_{50}=3.5$.

Using the correlated load combination approach, the total load, i.e., the combination load is $2.7(1.35+1.35=2.7)$ and its reliability is the same for each column, i.e. $\beta_{50}=3.5$, as the columns fail at the same time. 

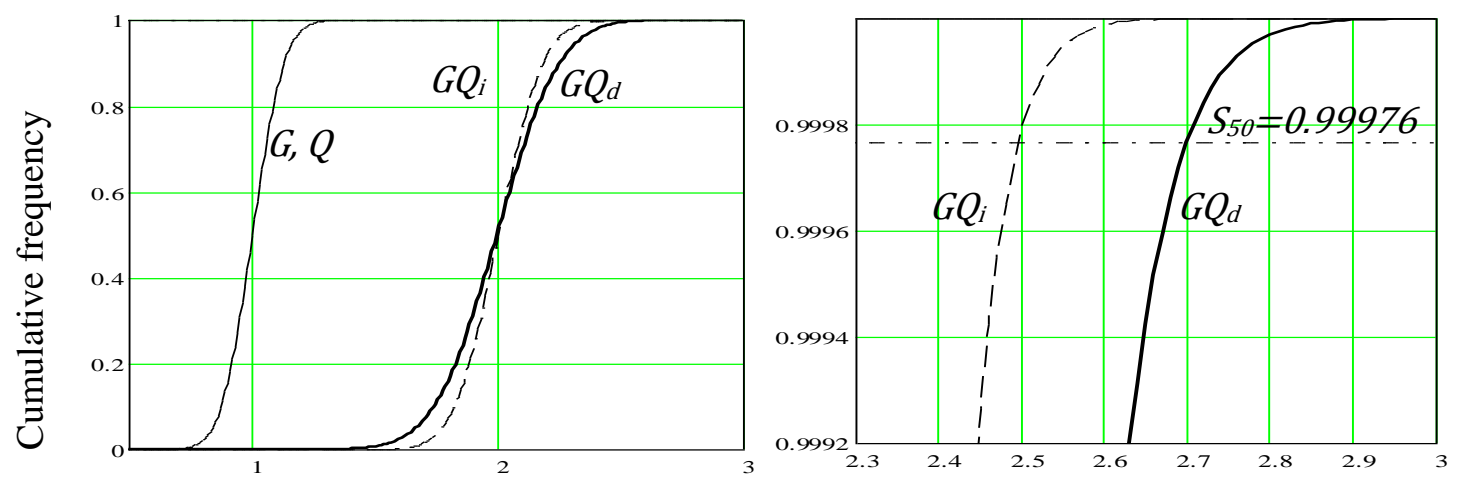

Figure 4. Distributions $G$ and $Q$, thin solid line, $G+Q$ combined correlated $G Q_{d}$ (thick solid line), $G+Q$ combined uncorrelated $G Q_{i}$ (dashed line). The target reliability is $\beta_{50}=3.5$, meaning that the survival probability is $S_{50}=0.9997674$ (horizontal dash-dotted line).

When the columns are combined (Figure 4 right), basic physics and statics unambiguously determine that the behaviour is equal for the two columns, i.e. the combined columns fail at the load 2.7, $\beta_{50}=3.5$.

However, if the loads are combined uncorrelated, the target reliability is reached at load 2.5 (2.495), which is ca $10 \%$ less than the actual strength. This is due to the load vanishing which occurs in the uncorrelated load combination. Consequently, the failure probability is $1 / 630$ of the correct one and corresponds to $\beta_{50}=4.95$. A graphic comparison between the correlated and uncorrelated combination approach has been included in Figure 4.

It is worthy of note that this example applies to all load proportions $\alpha$, with permanent and variable loads of $1.35 \alpha$ and $1.35(1-\alpha)$, respectively. The rules of statics demand that the combination load is 2.7 , i.e., the loads must be combined correlated in all load ratios.

\section{Statics}

The laws of statics demand that the combination load $g q$ of the actual permanent load $g$ and the variable load $q$ is $g+q$, which corresponds to the correlated combination. In the uncorrelated combination approach, it holds that $g+q<g q$ if the loads are high, i.e., a part of the load vanishes, and $g+q>g q$ if the loads are low, i.e., an excess load appears. Consequently, the statics viewpoint also suggests that the loads need to be combined correlated as the uncorrelated combination approach is in conflict with basic statics.

\section{An uncorrelated combination means correlated loads}

When the permanent load $g$ is combined uncorrelated with the variable load $q$ to obtain the combination load $g q$, the proportion of $g$ in $g q$ depends on both $g$ and $q$. Thus, in this combination $g$ and $q$ are correlated. For example, assume the permanent roof load $g$ produces action $a(g)$. In the uncorrelated load combination, when the snow load is added, $a(g)$ decreases (or sometimes increases), i.e., in this combination the loads are correlated because in the uncorrelated combination the partial loads become correlated in the 
combination process. Thus, the uncorrelated (non-simultaneous) combination cannot be used to combine uncorrelated loads.

\section{Further arguments}

With regard to the concepts of correlated and uncorrelated load combination, the current structural codes include several load combination contradictions, such as:

- The permanent and variable loads are normally combined uncorrelated for failure design (however, in some codes correlated), but in all codes, the loads are always combined correlated for serviceability design (e.g. deflection design). It is obvious that the load combination is equally correlated or uncorrelated in the failure and the serviceability design.

- The permanent loads of the floors in a multistorey house and parts of a structural component are always combined correlated, but the corresponding live loads are combined uncorrelated in the case of two live loads, but correlated in the case of several live loads. However, all these loads are uncorrelated, i.e. random and uncorrelated with each other. Thus, in the existing codes, the uncorrelated, random and uncorrelated loads do not consistently generate an uncorrelated combination.

Finally, we emphasize that the correlated combination approach, if used consistently, provides a solution to these contradictions.

\section{Discussion}

Uncorrelated load combination is likely to result in a lower reliability than is intended. In the Eurocodes, the theoretical insecure safety factor error is up to $14 \%$, which applies to the load case when the variable load is $20-30 \%$ of the total load, i.e., the load proportion $\alpha$ is $20-30 \%$. In this case, the 50-year failure probability $P_{f 50}$ is ca 40 times higher than intended, i.e., it is about $1 / 400\left(\beta_{f 50}=2.8\right)$. However, it should be $1 / 15000\left(\beta_{f 50}=3.8\right)$.

The example above shows that the actual error is lower, and the safety factor error is ca $10 \%$ lower in codes where the loads are combined uncorrelated. However, in some cases the actual error is higher: the material safety factors sometimes correspond to the correlated load combination and sometimes to the uncorrelated one. For example, this error occurs in the Finnish steel Eurocodes where the material safety factor is 1 . It is somewhat correct if the loads are combined correlated and the material safety factor is calculated uncorrelated. However, in the Finnish codes the loads are combined uncorrelated, i.e., the overall error is doubled to ca $20 \%$.

In the earlier codes, permanent and variable loads are combined correlated. However, in many of the codes published over recent decades, the uncorrelated load combination and the load reduction is adopted, e.g., in the Eurocodes, the combination rules (6.10a-b) and (6.10a, modified-b) are uncorrelated. The Eurocodes also include the correlated load combination rule (6.10), which is correct and should be used exclusively.

There is one important but ambiguous outcome: all design codes are based on safety factors defined by national code writers using undisclosed rules. No consensus procedure for setting the safety factor exists. For example, the Eurocodes lack clear instructions for safety factors. The current safety factors are apparently based sometimes on the 
uncorrelated load combination and sometimes on the correlated one. Therefore, the safety factors may include much the same error as does the load combination.

\section{Conclusion}

We propose that, for the safest possible outcome, the permanent load and the variable load should be combined without a reduction factor, i.e., correlated. In this respect, the combination rule (6.10) of the Eurocodes is correlated and correct, whereas the rules (6.10a-b) and (6.10a, modified-b), which use reduction factors, are uncorrelated and, therefore, also normally about $10 \%$ less safe and sometimes about $20 \%$ less safe.

The correlated combination approach, if used consistently, removes the load combination contradictions of the permanent and variable loads in the current codes.

\section{Acknowledgement}

Keijo Ruohonen, Professor of Mathematics at Tampere University of Technology has collaborated in the mathematical formulation of this paper. SP was supported by the Academy of Finland CoE in Inverse Problems.

\section{References}

[1] SFS-EN 1990:2006 Eurocode - Basis of structural design.

[2] Ferry Borges J., Castanheta M, 1971, Structural Safety, Laboratorio Nacional de Engenharia Civil, Lisbon

[3] ISO 2394, 1996, General principles on reliability for structures, International Organization for Standardization, Geneva, Switzerland.

[4] Walpole, R.E. \& Myers, R.H. \& Myers, S.L. \& Ye, K.: Probability \& Statistics for Engineers \& Scientists. Pearson (2012)

[5] Poutanen T., 2011, Calculation of partial safety factors, Applications of Statistics and Probability in Civil Engineering - Faber, Köhler \& Nishijima (eds), Taylor \& Francis Group, London

[6] Poutanen, T. 2013, Improved structural design code, ICOSSAR2013, New York

[7] Probabilistic Model Code, Part 1, Basis of Design, http://www.jcss.ethz.ch

[8] Guide to the Use of EN1990 Basis of Structural design, DCLG Publications, September 2006, www.communities.gov.uk

Tuomo Poutanen, Sampsa Pursiainen, Jari Mäkinen, Tim Länsivaara

Tampere University of Technology

Tekniikankatu 12

33101 Tampere 10

tuomo.poutanen@tut.fi, sampsa.pursiainen@tut.fi,jari.m.makinen@tut.fi, tim.lansivaara@tut.fi 